\title{
HIGH RESOLUTION OPTICAL IMAGING OF PROTO-PLANETARY NEBULAE
}

\author{
P.P. LANGILL and SUN KWOK \\ Dept. of Physics and Astronomy, University of Calgary, \\ Calgary, Alberta, Canada. T2N $1 N_{4}$ \\ and \\ B.J. HRIVNAK \\ Dept. of Physics, Valparaiso University, Valparaiso IN, USA. 46383
}

Several Proto-Planetary Nebulae (PPN) candidates have been observed at the Canada France Hawaii Telescope with the High Resolution Camera (HRCam) ( ${ }^{c}$ Clure et al. 1989, PASP, 101, 1156). Candidates were selected based on their IRAS [12]-[25] and [25]-[60] colors and their lack of variability. With HRCams' small angular pixel size (0.13"/pixel) and active optics we achieved an average angular resolution of 0.65 " in $\mathrm{V}$ and I waveband images.

We have determined standard V and I magnitudes for these PPN some of which had no previous optical counterpart. These measurements have allowed us to map the flux distributions of the PPN from the optical to the infrared. Measured distributions have been modeled by a detached dust shell radiative transfer code (Volk, K. and Kwok, S. 1989, Ap.J. 324, 345) which helps to determine parameters of the dust shell and where the PPN is in its evolution between the asymptotic giant branch phase and the planetary nebulae phase.

By observing in the optical we are able to study the light scattered from the dust in the PPN envelopes. With the high resoution achieved we are able to probe their morphologies. Comparing the PPN images to field star images we have determined that several PPN have extended dust shells. A few have also been found to be bipolar. The study of the morphology of PPN dust shells gives invaluable clues as to the initial conditions which lead to the large variety of PN morphologies (Balick, B. 1987, Ap.J. 94(3), 671). 